\title{
Fascin overexpression is regulated by the transactivation of the promoter but not by its hypomethylation in esophageal squamous cell carcinoma
}

\author{
JIAN HOU ${ }^{1,2^{*}}$, ZHANG-YAN GUO ${ }^{2 *}$, JIAN-JUN XIE $^{2}$, EN-MIN LI ${ }^{2}$ and LI-YAN XU ${ }^{1}$ \\ ${ }^{1}$ Institute of Oncologic Pathology, and ${ }^{2}$ Department of Biochemistry and Molecular Biology, \\ College of Medicine, Shantou University, Shantou 515041, P.R. China
}

Received February 16, 2009; Accepted June 29, 2009

DOI: 10.3892/mmr_00000182

\begin{abstract}
Fascin 1 (fascin) is known to be overexpressed in esophageal squamous cell carcinoma (ESCC); however, the mechanisms of this overexpression are unclear. In this study, the FSCN1 core promoter was isolated and the transcriptional regulatory mechanism of fascin overexpression in ESCC was investigated. By combining the use of progressive 5' deletions and dual-luciferase reporter assays, the FSCN1 core promoter was identified within the -74/-41 region in esophageal carcinoma EC109 cells harboring a GC box and a composite CRE/AP-1 binding site. Further analysis demonstrated that only the GC box was essential for transcription. No methylated $\mathrm{CpG}$ sites were found within the FSCN1 promoter in normal and tumor cells or tissues examined by methylation-specific PCR (MSP) and bisulfite genomic sequencing (BGS), which suggested that hypomethylation did not contribute to the overexpression of fascin in ESCC. Furthermore, the region of $+168 /+2838$ was found to inhibit promoter activity. Additional BGS analysis indicated that the region of $+560 /+859$ (in exon 1 ) was hypermethylated in normal and tumor cells or tissues. Unexpectedly, demethylation did not eliminate the suppression, suggesting that a silencer caused this suppression, and not DNA methylation in exon 1. Taken together, the results indicate that fascin overexpression in ESCC is regulated by the transactivation of the fascin promoter, but not by its hypomethylation.
\end{abstract}

Correspondence to: Dr En-Min Li, Department of Biochemistry and Molecular Biology, College of Medicine, Shantou University, Shantou 515041, P.R. China

E-mail:nmli@stu.edu.cn

Dr Li-Yan Xu, Institute of Oncologic Pathology, College of Medicine, Shantou University, Shantou 515041, P.R. China

E-mail: liyanxu1130@yahoo.com.cn

${ }^{*}$ Contributed equally

Key words: fascin 1 gene, promoter, transactivation, methylation, esophageal squamous cell carcinoma

\section{Introduction}

Fascin 1 (fascin), a 55-kDa globular actin-bundling protein (1), is expressed in specific tissues and cells (2). In tissues such as brain and spleen, fascin is abundantly expressed, while at the cellular level it is observed mainly in neuronal and glial cells and microcapillary endothelial cells, as well as in antigenpresenting dendritic cells (DCs) (3-6).

Fascin is expressed at low levels or is absent in normal epithelia, but is overexpressed in many human carcinomas (7). Fascin overexpression is correlated with poor patient prognosis in certain tumors, such as non-small-cell lung cancer and gastric, breast and oesophageal carcinomas (8-11). In esophageal squamous cell carcinoma (ESCC), it has been shown that the up-regulation of fascin occurs during the early stages of malignant transformation and is correlated with lymph-node metastasis (12). Hashimoto et al reported that in ESCC the infiltrative margins tended to stain most strongly for fascin (11), while a report from Xie et al revealed that fascin was localized in the membrane protrusion of ESCC cells, and that the down-regulation of fascin resulted in the suppression of cell proliferation and invasiveness (13). These findings strongly suggest that the overexpression of fascin plays an important role in invasion and metastasis in ESCC. However, the mechanisms by which fascin is up-regulated in carcinoma cells remain unclear.

Human fascin is encoded by the FSCN1 gene located at chromosome 7p22, which contains a 9-kb-intron 1 and another three introns. FSCN1 mRNA consists of a 121-bp 5' untranslated region, a 1174-bp 3' untranslated region and a 1482-bp coding region producing a 493 amino acid polypeptide. Bros et al demonstrated that the core promoter of FSCN1 is located 100-bp upstream from the transcription start site in DCs, and that this region contains a putative GC box, a composite CRE/ AP-1 binding site and a TATA box (14). However, it is not yet known whether the same mechanism of transcriptional regulation is utilized in carcinoma cells.

Dense $\mathrm{CpG}$ sites in promoter or exon/intron regions are usually indicative of potential transcriptional regulatory elements. Methylation of these $\mathrm{CpG}$ sites blocks the binding of regulatory proteins, resulting in transcription repression (15). CpG island analysis using the CpGPLot program (EBI, 
Table I. Sequences of primers used.

\begin{tabular}{|c|c|c|}
\hline PCR & Primers & Location (AY044229) \\
\hline \multicolumn{3}{|c|}{ MSP for FSCN1 promoter } \\
\hline FsnMF2 & 5'-GTGGCGTTGACGTTATTTC-3' & -56 to -38 \\
\hline FsnMR2 & 5'-ATAAACGAAAAACCGCTACG-3' & +95 to +114 \\
\hline FsnUF2 & 5'-TTGGTGGTGTTGATGTTATTTT-3' & -59 to -38 \\
\hline FsnUR2 & 5'-ATAAACAAAAAACCACTACACAA-3' & +92 to +114 \\
\hline \multicolumn{3}{|c|}{ BGS for FSCN1 promoter } \\
\hline FsnBGS1 & 5'-GTGAGGGGTGATGTTTTT-3' & -218 to -199 \\
\hline FsnBGS2 & 5'-CCCTACTCCTTTATTCRACTAC-3' & +34 to +55 \\
\hline \multicolumn{3}{|c|}{ BGS for FSCN1 exon 1} \\
\hline 261-BGSF & 5'-GTTTGAAGAAGAAGTAGATTTGGA-3' & +237 to +260 \\
\hline 261-BGSR & 5'-TAACGATATACACGCTCCACT-3' & +493 to +513 \\
\hline 638-BGSF & 5'-AGCGTTACGCGTATTTGAG-3' & +560 to +578 \\
\hline 638-BGSR & 5'-ТАСCCACCTTAATAACCTTACCC-3' & +837 to +859 \\
\hline
\end{tabular}

http://www.ebi.ac.uk/emboss/cpgplot) revealed that the FSCN1 promoter region was highly GC-rich $(73.86 \%)$, with a $\mathrm{CpG}$ island located at $-270 /+130$. Two $\mathrm{CpG}$ islands were also found at exon 1 and intron $1(+216 /+1127$ and $+1330 /+2151)$, suggesting the presence of regulatory elements. To investigate the transcriptional regulation mechanism of FSCN1 in ESCC, the core promoter of FSCN1 was isolated from EC109 cells and an ESCC cell line (16), and the role of the FSCN1 extron 1/ intron 1 as a regulatory region was examined by methylation analysis.

\section{Materials and methods}

Reporter gene constructs. To define the extension of the FSCN1 promoter, a 3022-bp sequence $(-2900 /+122)$ including the major part of the 5'-flanking region of FSCN1 (accession no. AY044229) was amplified, cloned into the pGL3-Basic vector (Promega, Madison, WI, USA) and named pBF-2900. The following primers were used: 5'-CAG TCAAGACCTAGCACAGGGCTCAGG-3' and 5'-GGTGGC AGTAGACGAGAGGCCGCTG-3'. A series of 5' deletion constructs of pBF-2900 was obtained using the Erase-a-Base ${ }^{\circledR}$ System (Promega). To compare regulatory elements within the promoter in ESCC cells with those in DCs, relevant restriction sites (AatII, Aat II/NheI, AatII/ApaI and ApaI/NheI) in the core promoter region were examined, and four constructs with small internal deletions were identified (14).

To assess the regulatory activity of exon 1/intron 1 , a corresponding fragment $2671 \mathrm{bp}$ in length $(+168 /+2838)$ was cloned into the pGL3-Promoter vector (pGLP) of Promega (named pP/Fi2671) and pBF-74 (named pBF-74/Fi2671), respectively, containing the SV40 promoter or the FSCN1 core promoter. Two deletion constructs, pBF-74/Fi628 and pBF-74/ Fi261, were obtained by digesting pBF-74/Fi2671 plasmid with SalI and $S a l I / B g l I I$, respectively.

Cell culture and transfection. The human esophageal carcinoma cell line EC109 was cultured in 199 medium (Invitrogen,
Carlsbad, CA, USA) containing 10\% fetal bovine serum in an atmosphere of $5 \% \mathrm{CO}_{2}$ at $37^{\circ} \mathrm{C}$. For transfection, cells were plated in 96 -well plates at $1.5 \times 10^{5}$ cells $/ \mathrm{ml}$, grown to $50-80 \%$ confluence and transfected with the plasmids described above using Superfect Transfection Reagent (Qiagen, Maryland, MD, USA) according to the manufacturer's instructions. Cells were treated with $10 \mu \mathrm{M}$ 5-aza-dC (Sigma-Aldrich, St. Louis, MO, USA) for another $48 \mathrm{~h}$ before being harvested for the luciferase assay.

Luciferase assay. Cells transfected with luciferase constructs were harvested and lysed in Passive Lysis Buffer (Promega) after $48 \mathrm{~h}$. The cell lysates were analyzed for firefly and renilla luciferase activity using the Dual-Luciferase Reporter Assay System (Promega) as recommended by the manufacturer. Firefly luciferase activity was normalized to renilla luciferase activity as an internal control.

Bisulfite treatment, methylation-specific PCR and bisulfite genomic sequencing. Several cell lines were used, including esophageal carcinoma cell lines such as EC18 (also known as EC/CUHK2; provided by Professor Z.Y. Shen of the Medical College of Shantou University, Shantou, P.R. China), EC109, EC8712, SHEEC $(17,18)$ and an immortalized esophageal epithelial cell line, SHEE (19). These cell lines were routinely cultured in 199 medium. Eighteen primary tumors and their corresponding normal tissues were obtained from the Pathology Department of the Medical College of Shantou University. Genomic DNA was extracted from cell pellets or tissues. $10 \mu \mathrm{g}$ of the DNA was denatured by the addition of $3.3 \mu \mathrm{l}$ of $3 \mathrm{M} \mathrm{NaOH}$ at $37^{\circ} \mathrm{C}$ for $15 \mathrm{~min}$. Subsequently, $333 \mu \mathrm{l}$ of bisulfite solution was added and the DNA was incubated in darkness for $4 \mathrm{~h}$ at $55^{\circ} \mathrm{C}$. The modified DNA was purified using the Wizard DNA Clean-Up System (Promega) and dissolved in TE buffer (final concentration, $1 \mu \mathrm{g} / 20 \mu \mathrm{l}$ ).

For methylation-specific PCR (MSP), treated DNA was amplified using a methylation-specific primer or an unmethylation-specific primer set (Table I). MSP was performed in 
A.

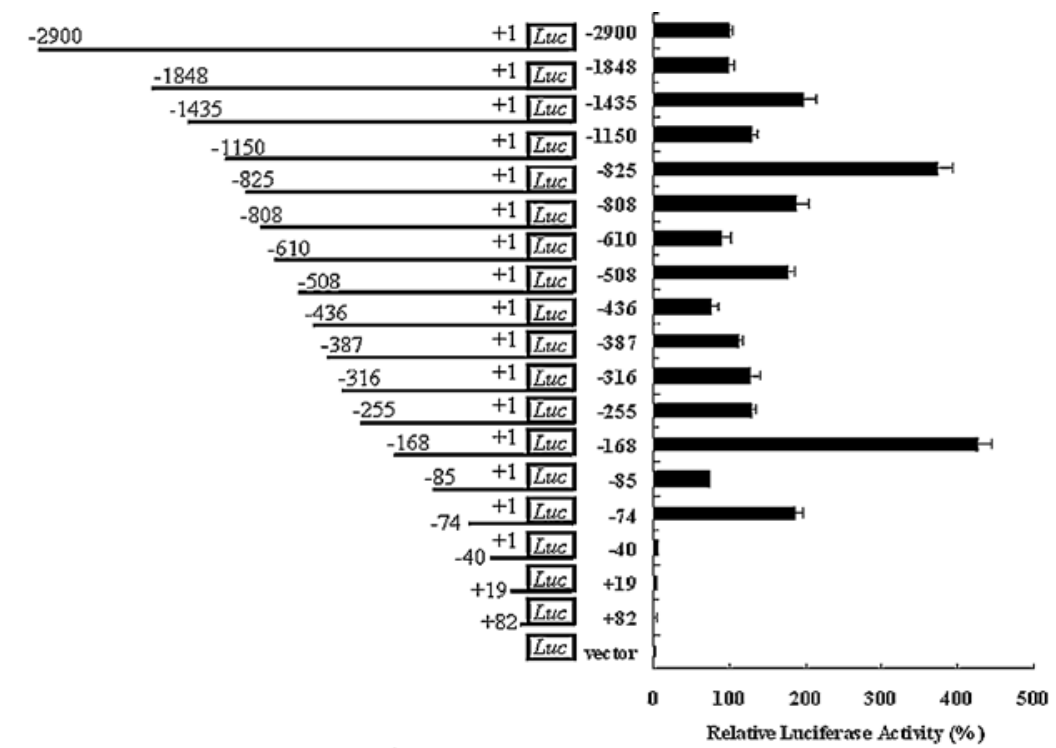

B.

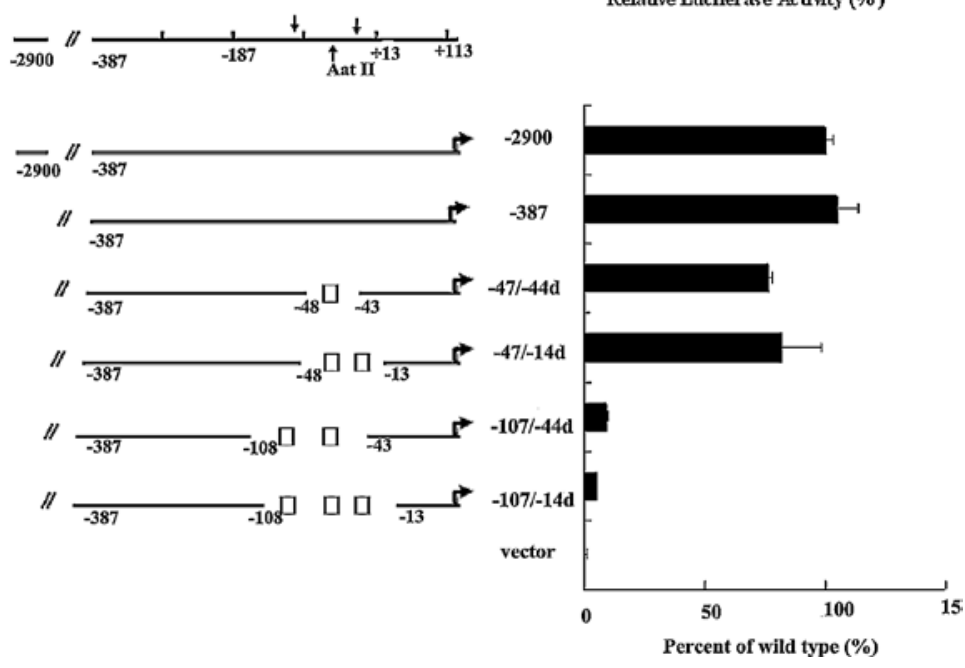

Figure 1. Identification of the core promoter of human FSCN1 in EC109 cells. EC109 cells were transiently transfected with luciferase reporter constructs, and reporter activity was detected by dual-luciferase assay. (A) Localization of the transcriptional regulatory region of human FSCN1 by 5'-deletion analysis. (B) Identification of the regulatory element within the human FSCN1 core promoter. pBF-387 was modified by a small internal deletion. The regions of $-47 /-44$ (CREB/AP-1 site), -47/-14 (CREB/AP-1 and TATA box), -107/-44 (GC box and CREB/AP-1 site) and -107/-14 (GC box, CREB/AP-1 and TATA box) were deleted. The activity of the deletion constructs was relative to that of pBF-2900. pGL3-Basic vector (vector) served as a blank control. Each value represents the mean \pm SD. Data are representative of at least two independent experiments. Transfections were carried out in triplicate for each experiment.

40 cycles of $94^{\circ} \mathrm{C}$ for $30 \mathrm{sec}, 58^{\circ} \mathrm{C}$ for $30 \mathrm{sec}$ and $72^{\circ} \mathrm{C}$ for $30 \mathrm{sec}$ using the AmpliTaq Gold system (Applied Biosystems, Foster City, CA, USA). For bisulfite genomic sequencing (BGS), modified DNA was amplified using several BGS primer sets (Table I) to analyze the methylation status of the FSCN1 promoter and exon 1. BGS was performed in 40 cycles of $94^{\circ} \mathrm{C}$ for $30 \mathrm{sec}, 50^{\circ} \mathrm{C}$ for $30 \mathrm{sec}$ and $72^{\circ} \mathrm{C}$ for $30 \mathrm{sec}$ using HotStarTaq DNA Polymerase (Qiagen). The PCR products were cloned into pGEM $^{\circledR}$-T Easy Vector (Promega). At least three clones were randomly chosen and sequenced.

\section{Results}

The specific core promoter of human FSCN1 isolated from EC109 cells contained an essential GC box. To define the extension of the FSCN1 promoter in EC109 cells, a series of constructs was obtained by 5'-deletion experiments from pBF-2900 plasmid, and then analyzed with the Dual-Luciferase
Reporter Assay System. Compared to pBF-2900, the activity of the deletion constructs from pBF-1848 to pBF-74 was generally $>80 \%$, while the 5'-deletion from -74 to -41 abolished most activity (Fig. 1A), suggesting that the $-74 /-41$ region was the core promoter of the FSCN1 (the promoter region in EC109 cells: accession number EU486847) in EC109 cells. The region of $-100 /+1$, which is highly conserved in humans and mice, contains a TATA box, a GC box and a composite CRE/AP-1 binding site (14). Internal deletion analysis was performed in the core promoter region to identify the regulatory elements. As shown in Fig. 1B, deletion of the -47/-44 segment (CRE/ AP-1 site) or the -47/-14 segment (CRE/AP-1 and TATA box) did not significantly alter reporter activity. However, reporter activity decreased significantly when the -107/-44 segment (GC box and CRE/AP-1 site) or the -107/-14 segment (GC box, CRE/AP-1 site and TATA box) was deleted, indicating that the GC box, rather than the TATA box, is essential for FSCN1 transcription in EC109 cells. 
A.

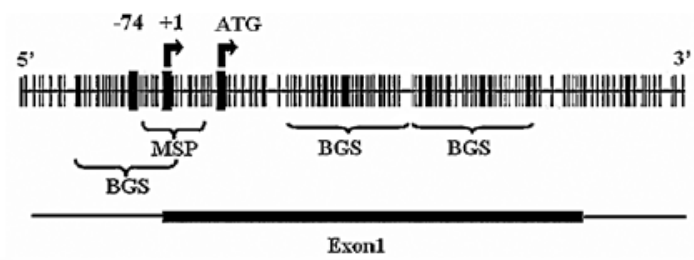

B.

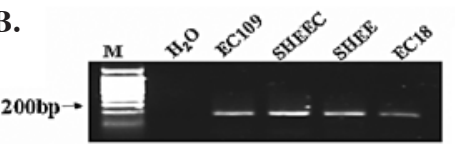

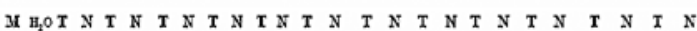

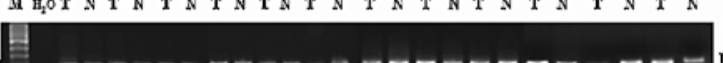

D.

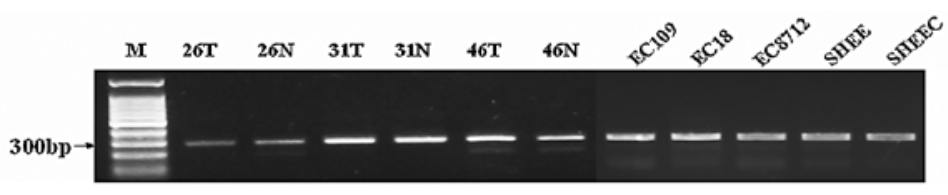

E.

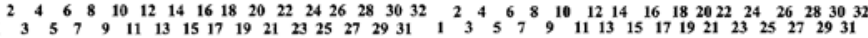

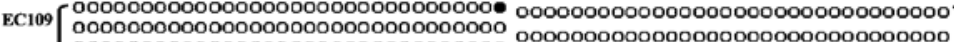
s 00000000000000000000000000000000 EC18 $0000000000000 \Delta 0000000$ (c8712 00000000000000000000000000000000 00000000000000000000000000000000 0000000000000000000000000000000

F.

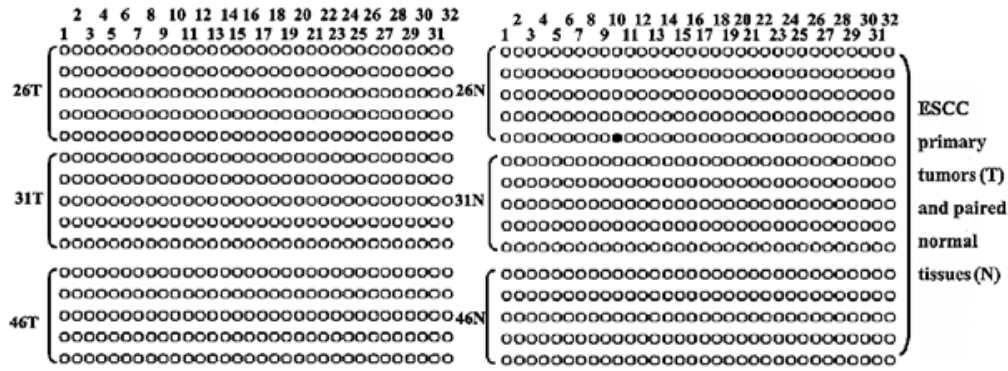

Figure 2. Hypomethylation of FSCN1 promoter in ESCC. (A) Schematic structure of the FSCN1 promoter and exon 1: exon 1, dark rectangle; CpG sites in the $\mathrm{CpG}$ island, short vertical lines; transcription start site and translation initiation site, curved arrows. The MSP and BGS regions are also indicated. (B) Representative MSP results for ESCC cells and immortalized SHEE cells. (C) Representative MSP results for ESCC primary tumors (T) and corresponding normal tissues $(\mathrm{N})$. Only unmethylated bands were amplified in cells or tissues. U, unmethylated. (D,E and F) Representative high-resolution analysis of the methylation status of individual CpG sites in the FSCN1 promoter by BGS in cell lines and primary tumors. Each circle corresponds to a single CpG site. Methylated sites are indicated by filled dark circles and unmethylated sites by empty white ones. $\Delta$, not tested.

No methylated sites in the FSCN1 promoter in ESCC. As described above, many $\mathrm{CpG}$ sites are present in the FSCN1 promoter (Fig. 2A), suggesting that methylation may regulate the transcription of FSCN1. MSP and BGS experiments were used to analyze the methylation status of the FSCN1 promoter. Using MSP, methylated alleles were detected in the ESCC cell lines and in the SHEE immortalized esophageal epithelial cell line, as well as in 18 primary tumors and their corresponding normal tissues. However, in all samples mentioned above, only the unmethylated band was amplified (Fig. 2B and C; data from methylation reaction not shown). BGS analyses confirmed these results (Fig. 2D, E and F), suggesting that hypomethylation does not contribute to fascin up-regulation in ESCC.

Promoter activity was suppressed by an inhibitory element in exon 1/intron 1, but not by the methylation of exon 1 . Bioinformatics analysis revealed that FSCN1 exon 1/intron 1 was characterized by an unusually high GC content and contained two $\mathrm{CpG}$ islands, suggesting the presence of methylated sites regulating promoter activity. To investigate this possibility, two reporter constructs with the FSCN1 2671-bp fragment containing exon 1 and part of intron 1 were generated by cloning into the downstream of the luciferase polyadenylation signal of the reporter plasmid pBF-74 and the pGLP vector (named pBF-74/Fi2671 and pP/Fi2671, respectively). These constructs were then transfected into EC109 cells, and reporter activity was measured. Of note, when EC109 cells were tested, a significant decrease in reporter activity was observed (Fig. 3A), suggesting that this cloned region may have a suppressive function. Such a suppressor region might regulate the activities of the FSCN1 and SV40 promoters.

To evaluate whether methylation of this region regulated promoter activity, two segments $(+237 /+513$ and $+560 /+859)$ in the region were analyzed by BGS (Fig. 2A). The results showed 
A.

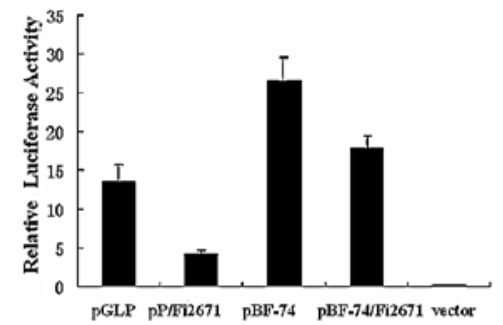

C.

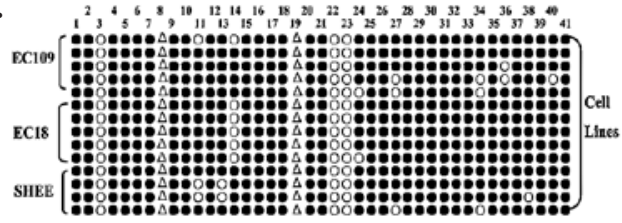

E.

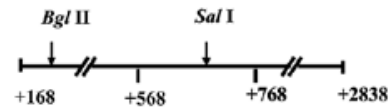

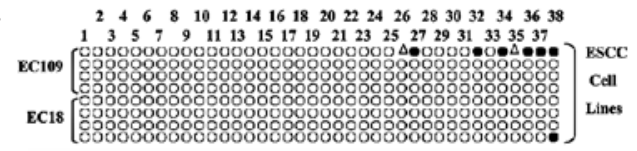

D.

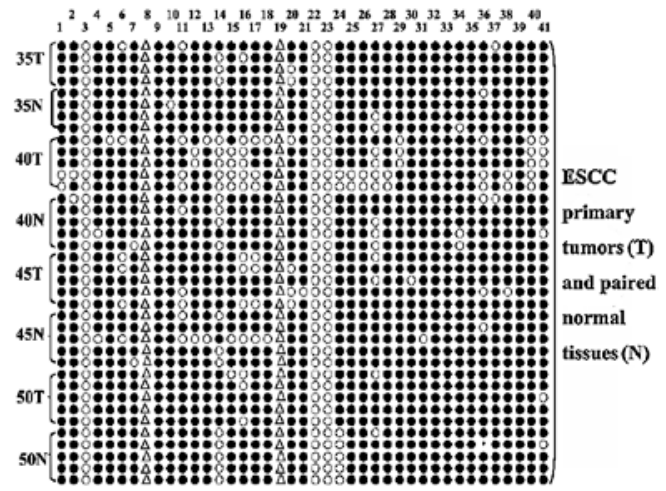

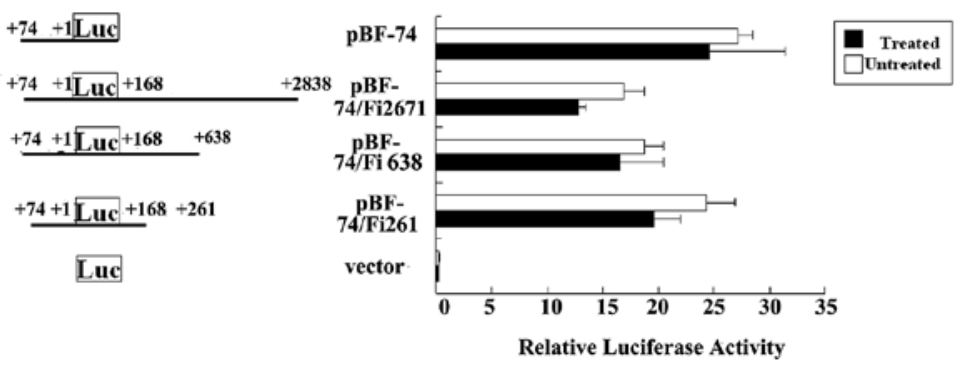

Figure 3. Cis-regulatory element in FSCN1 exon 1/intron 1. (A) The effect of FSCN1 exon 1/intron on the activity of the FSCN1 core promoter and SV40 promoter. (B) BGS analysis of $38 \mathrm{CpG}$ sites in the region $+237 /+513$ of FSCN1 in the cell lines. (C and D) BGS analysis of $41 \mathrm{CpG}$ sites in the region $+560 /+829$ of FSCN1 in the cell lines, ESCC primary tumors (T) and corresponding normal tissues (N). (E) Analysis of FSCN1 exon 1/intron 1 activity in EC109 after treatment with 5-aza-dC. Each value represents the mean $\pm \mathrm{SD}$. Data are representative of at least two independent experiments. Transfections were carried out in triplicate for each experiment. $\Delta$, not tested.

that the region of $+560 /+859$ (in exon 1 ) was hypermethylated in all samples, including the ESCC cell lines, an immortalized SHEE cell line, and five primary tumors and their corresponding normal tissues (Fig. 3C and D); however, no methylation sites were found in the $+237 /+513$ region (Fig. 3B). Further experiments to address whether methylation in exon 1 could lead to suppression were performed with the luciferase reporter assay. Several reporter plasmids were constructed by inserting various parts of a 2671-bp fragment into the reporter plasmid pBF-74. After transfection, the EC109 cells were treated with 5-aza-dC, a specific demethylating agent, for $48 \mathrm{~h}$ to assess whether demethylation eliminated suppression. The reporter activity of the untreated group showed that the deletion from +639 to +2838 caused a moderate increase in activity compared to $\mathrm{pBF}-74 / \mathrm{Fi} 2671$, and that the deletion from +262 to +2838 resulted in similar activity as the basal promoter $(95 \%$ of the basal promoter), suggesting that this region may function as an inhibitory region. Unexpectedly, the treated cells showed almost the same level of luciferase activity for each construct as the untreated cells (Fig. 3E), indicating that the suppression was correlated with a silencer in exon 1/intron 1 rather than with localized methylation in exon 1 .

\section{Discussion}

Fascin is expressed at low levels or is absent in normal epithelia, but is up-regulated at translational and transcriptional levels in ESCC (12). Overexpression of fascin is an early event in tumor progression and is correlated with tumor invasion and poor prognosis in ESCC $(11,12)$. Previous reports suggest that fascin may be a novel candidate biomarker or therapeutic target for ESCC (7). Thus, elucidation of the mechanisms underlying the regulated expression of fascin may provide important information regarding its use as a potential marker or therapeutic target.

The present data suggest novel mechanisms underlying the regulation of FSCN1 transcription in ESCC. It was previously reported that, in DCs, the core promoter of FSCN1 contained a putative GC box, a composite CRE/AP-1 binding site and a TATA box. Internal deletion of the TATA box resulted in a significant reduction of reporter activity compared with the full-length promoter (14). Notably, this study shows that, in EC109 cells, the core promoter of FSCN1 was located upstream of the TATA box. When the TATA box and/or the CRE/AP-1 binding site were deleted, the activity did not alter remarkably. 
However, the deletion of the GC box affected promoter activity severely. This indicates that the GC box, rather than the TATA box, was essential for FSCN1 transcription in EC109 cells (Fig. 1B). Thus, the FSCN1 transcription regulatory mechanism in EC109 cells may be distinguished from that in DCs. High expression of fascin in carcinoma cells and in DCs may be mediated by different signaling pathways, resulting in tissue-specific cis-acting elements of the promoter. The GC box is recognized by members of the Sp/XKLF family (Sp1, Sp3, Sp4, TIEG2 and BTEB1) (20). As transcription factors, the $\mathrm{Sp}$ family is characterized by the highly conserved Cys2His2 zinc-finger DNA binding domain at the C-termini, and all members of the Sp protein family belong to the Kruppel-like zinc-finger superfamily (21-23). Among the Sp family, Sp1 is widely expressed in different tissues and can bind to GC box motifs in promoters. Phosphorylation of Sp1 by many kinases can result in functional changes, such as altered DNA binding and/or promoter activation (24). Further studies are required to elucidate the relationship between FSCN1 transcription and Sp1 function.

Decreased methylation levels of oncogenes are common in human tumors (15). Several studies have revealed that there is a good inverse correlation between methylation and gene expression. Overexpression of P450 1B1 was regulated by hypomethylation in prostate cancer (25), while P-cadherin mRNA expression in invasive breast carcinomas was associated with the hypomethylation of the $\mathrm{CDH} 3$ promoter (26). These reports suggest that the hypomethylation of specific oncogene promoters may play an important role in tumorigenesis. Bioinformatics analysis revealed that the FSCN1 promoter was highly GC-rich and contained a potential $\mathrm{CpG}$ island. However, the data in this study showed that the $\mathrm{CpG}$ sites within the FSCN1 promoter were unmethylated in normal and tumor cells or tissues. These results suggest that hypomethylation of the promoter is not involved in fascin overexpression in ESCC. In short, overexpression of fascin in ESCC may result from the transactivation of a transcriptional factor, but not from its hypomethylation.

Cis-acting enhancer/suppressor elements are frequently located within exons and introns and regulate promoter activity. The exon 1/intron 1 of FSCN1 is characterized by an unusually high $\mathrm{GC}$ content, and contains two $\mathrm{CpG}$ islands. The results of this study revealed that FSCN1 exon 1/intron 1 may function as a silencer. BGS analysis indicated that the region of $+560 /+859$ (in exon 1) was hypermethylated in an immortalized SHEE cell line, two ESCC cell lines and five primary tumors with their corresponding normal tissues, suggesting that the inhibition may be caused by the hypermethylation within exon 1 . Recent reports suggested that hypermethylation in an exon/intron to influenced promoter activity in other genes. Hypermethylation of IGFBBP7 within exon 1 was closely related to the absence of its expression in colon cancer (27). Aberrant vimentin exon 1 methylation was correlated with vimentin gene overexpression in colon cancer (28). Methylation within exons of the BCSG1 gene could silence its expression in breast tissue (29). Methylation at $\mathrm{CpG}$ islands in intron 1 of EGR2 conferred enhancer-like activity (30). Unexpectedly, our further demethylation experiments did not support this hypothesis, since demethylation did not eliminate the suppression of FSCN1 exon 1/intron 1. Therefore, the suppression may be mediated by an unknown silencer, but not by the methylation at $\mathrm{CpG}$ islands within exon 1 . Nonetheless, this suppressor region could not inhibit promoter activity completely. Once a strong signaling pathway is activated, the promoter can disrupt suppression, thereby allowing FSCN1 to be transcribed. Thus, it is more significant to identify the activated signaling pathway and the transcription factor in ESCC.

In conclusion, the FSCN1 core promoter is located in the $-74 /-40$ region and the GC box is essential for FSCN1 transcription in EC109 cells. Overexpression of fascin in ESCC is regulated by transactivation of the promoter, but not its hypomethylation. The inhibitory element in exon 1/intron 1 may suppress the promoter activity to an extent, but not completely.

\section{Acknowledgements}

This work was supported by grants from the National High Technology Research and Development Program of China (2006AA02A403), the National Natural Science Foundation of China (30672376 and 30772485) and the Guangdong Scientific Fund for Key Items (5104541 and 7118419).

\section{References}

1. Edwards RA and Bryan J: Fascins, a family of actin bundling proteins. Cell Motil Cytoskel 32: 1-9, 1995.

2. Zhang FR, Tao LH, Shen ZY, Lv Z, Xu LY and Li EM: Fascin expression in human embryonic, fetal, and normal adult tissue. J Histochem Cytochem 56: 193-199, 2008.

3. Duh FM, Latif F, Weng Y, Geil L, Modi W and Stackhouse T: cDNA cloning and expression of the human homolog of the sea urchin fascin and Drosophila singed genes which encodes an actin-bundling protein. DNA Cell Biol 13: 821-827, 1994.

4. Mosialos G, Yamashiro S, Baughman RW, Matsudaira P, Vara L and Matsumura F: Epstein-Barr virus infection induces expression in B lymphocytes of a novel gene encoding an evolutionarily conserved 55-kilodalton actin-bundling protein. J Virol 68: 7320-7328, 1994.

5. Mosialos G, Birkenbach M, Ayehunie S, Matsumura F, Pinkus GS and Kieff E: Circulating human dendritic cells differentially express high levels of a 55-kDa actin-bundling protein. Am J Pathol 148: 593-600, 1996.

6. Pinkus GS, Pinkus JL, Langhoff E, Matsumura F, Yamashiro $\mathrm{S}$ and Mosialos G: Fascin, a sensitive new marker for ReedSternberg cells of Hodgkin's disease. Evidence for a dendritic or B cell derivation? Am J Pathol 150: 543-562, 1997.

7. Hashimoto Y, Skacel M and Adams JC: Roles of fascin in human carcinoma motility and signaling: prospects for a novel biomarker? Int J Biochem Cell Biol 37: 1787-1804, 2005.

8. Pelosi G, Pastorino U, Pasini F, Maissoneuve P, Fraggetta F and Iannucci A: Independent prognostic value of fascin immunoreactivity in stage I non-small cell lung cancer. $\mathrm{Br} \mathrm{J}$ Cancer 88: 537-547, 2003.

9. Hashimoto Y, Shimada Y, Kawamura J, Yamasaki S and Imamura M: The prognostic relevance of fascin expression in human gastric carcinoma. Oncology 67: 262-270, 2004.

10. Yoder BJ, Tso E, Skacel M, Pettay J, Tarr S and Budd T: The expression of fascin, an actin-bundling motility protein, correlates with hormone receptor-negative breast cancer and a more aggressive clinical course. Clin Cancer Res 11: 186-192, 2005.

11. Hashimoto $Y$, Ito $T$, Inoue $H$, Okumura $T$, Tanaka $E$ and Tsunoda S: Prognostic significance of fascin overexpression in human esophageal squamous cell carcinoma. Clin Cancer Res 11: 2597-2605, 2005.

12. Zhang H, Xu L, Xiao D, Xie J, Zeng H and Cai W: Fascin is a potential biomarker for early-stage oesophageal squamous cell carcinoma. J Clin Pathol 59: 958-964, 2006.

13. Xie JJ, Xu LY, Zhang HH, Cai WJ, Mai RQ and Xie YM: Role of fascin in the proliferation and invasiveness of esophageal carcinoma cells. Biochem Biophys Res Commun 337: 355-362, 2005. 
14. Bros M, Ross XL, Pautz A, Reske-Kunz AB and Ross R: The human fascin gene promoter is highly active in mature dendritic cells due to a stage-specific enhancer. J Immunol 171: 1825-1834, 2003.

15. Singal R and Ginder GD: DNAMethylation. Blood 93: 4059-4070, 1999.

16. Pan Q and Xue X: The proliferation and cell cycle of a human epithelial cell line (Eca 109) from esophageal carcinoma. J Mol Cell Biol 13: 19-27, 1890.

17. Wang X, Xiao F, Wang M, Zhou C and Wu M: Establishment of two human esophageal carcinoma cell lines and their cytogenetic analysis. Chin J Oncol 20: 3-6, 1998.

18. Shen Z, Cai W and Shen J: Human papilloma virus 18E6E7 in synergy with TPA induced malignant transformation of human embryonic esophageal epithelial cells. Chin J Virol 15: 5-10, 100-101, 1999.

19. Shen Z, Cen S and Cai W: Immortalization of human fetal esophageal epithelial cells induced by E6 and E7 genes of human papilloma virus 18. Chin J Exp Clin Virol 13: 121-124, 1999.

20. Li L, He S, Sun JM and Davie JR: Gene regulation by Sp1 and Sp3. Biochem Cell Biol 82: 460-471, 2004.

21. Kaczynski J, Cook T and Urrutia R: Sp1- and Krüppel-like transcription factors. Genome Biol 4: 206-208, 2003.

22. Bouwman P and Philipsen S: Regulation of the activity of Sp1related transcription factors. Mol Cell Endocrinol 195: 27-38, 2002.

23. Suske G: The Sp-family of transcription factors. Gene 238: 291-300, 1999.
24. Chu S and Ferro TJ: Sp1: Regulation of gene expression by phosphorylation. Gene 348: 1-11, 2005.

25. Tokizane T, Shiina H and Igawa M: Cytochrome P450 1B1 is overexpressed and regulated by hypomethylation in prostate cancer. Clin Cancer Res 11: 5793-5801, 2005.

26. Paredes J, Albergaria A, Oliveira JT, Jeronimo C, Milanezi F and Schmitt FC: P-cadherin overexpression is an indicator of clinical outcome in invasive breast carcinomas and is associated with $\mathrm{CDH} 3$ promoter hypomethylation. Clin Cancer Res 11: 5869-5877, 2005

27. Lin J, Lai M, Huang Q, Ma Y, Cui J and Ruan W: Methylation patterns of IGFBP7 in colon cancer cell lines are associated with levels of gene expression. J Pathol 212: 83-90, 2007.

28. Chen WD, Han ZJ, Skoletsky J, Olson J, Sah J and Myeroff L: Detection in fecal DNA of colon cancer-specific methylation of the nonexpressed vimentin gene. J Natl Cancer Inst 97: 1124-1132, 2005.

29. Lu A, Gupta A, Li C, Ahlborn TE, Ma Y and Shi EY: Molecular mechanisms for aberrant expression of the human breast cancer specific gene 1 in breast cancer cells: control of transcription by DNA methylation and intronic sequences. Oncogene 20: 5173-5185, 2001.

30. Unoki $\mathrm{M}$ and Nakamura $\mathrm{Y}$ : Methylation at $\mathrm{CpG}$ islands in intron 1 of EGR2 confers enhancer-like activity. FEBS Lett 554: 67-72, 2003. 